\title{
Neighbour wireless body area network discovery mechanism for ETSI SmartBAN
}

\author{
Tuomas Paso and Jussi Haapola \\ University of Oulu, Centre for Wireless Communications, P.O. Box 4500, 90014-Oulu, \\ FINLAND \\ tuomas.paso@oulu.fi,jussi.haapola@oulu.fi
}

\begin{abstract}
A novel neighbour network discovery mechanism targeted especially for wireless body area networks (WBANs) is defined and analysed in this paper. The Institute of Electrical and Electronics Engineers (IEEE) standard 802.15.6 and the European Telecommunications Standards Institute (ETSI) technical committee SmartBAN define physical (PHY) and medium access control (MAC) specifications for packet-based short-range communications in WBANs. Neither the fore-mentioned standards provide a solution for discovery of operating WBAN networks by another operating WBAN using the same standard specifications in near vicinity. The proposed method provides a SmartBAN compliant mechanism to discover other neighbouring SmartBAN networks while maintaining uninterrupted operations of both the wireless network carrying out the discovery and the wireless networks to be discovered. A SmartBAN network operates in two physical channels: a control channel $(\mathrm{CCH})$ and a data channel $(\mathrm{DCH})$. The key feature of the mechanism is the utilisation of the Inactive Periods of the discovering SmartBAN's DCH by alternating the Inactive Period durations for scanning CCH Beacons of the target SmartBAN. Based on the analysis, it can be concluded the proposed mechanism outperforms a mechanism in which the scan is performed in a fixed cyclical mode in terms of probability of discovery and discovery time. Currently, the discovery mechanism is under consideration for the revision of MAC spefications in TC SmartBAN.
\end{abstract}

Keywords: Medium Access Contol (MAC), Neighbour Network Discovery, Wireless Body Area Network (WBAN).

\section{Introduction}

The Institute of Electrical and Electronics Engineers (IEEE) standard 802.15.6 [1] and The European Telecommunications Standards Institute (ETSI) technical committee SmartBAN [2], [3] define physical (PHY) and medium access control (MAC) specifications for packet-based short-range communications in wireless body area networks (WBANs). WBANs target, e.g. medical and healthcare monitoring systems in the vicinity of, or inside a human body.

Discovery of neighbour WBAN networks is the first step in enabling inter-WBAN communications. However, the fore-mentioned IEEE and the ETSI standards provide 
only hooks to implement provider specific means for detection of existence and coexistence with other neighbour networks, but do not specify mechanisms for discovery using the same standard specifications in near vicinity. Wireless sensor network research has identified over the years various mechanisms for neighbour network discovery. Such mechanisms can roughly be classified in four underlying principles: randomness, over-half occupation, rotation-resistant intersection, and coprime cycles [4]. For Bluetooth Low Energy devices, a precision mechanism has been proposed in [5] and [6], in which the devices can operate in three different modes, i.e., advertising, scanning, and initiating. However, the proposed mechanisms focus on the case where a new device initialises and attempts to discover an existing network.

The contribution of this paper is the proposal of a mechanism for the case where a WBAN is already operational and attempts to discover another operational WBAN without interrupting either WBAN operations already being carried out. The mechanism is SmartBAN compliant, and currently it is under consideration to be included in the ongoing revision of the SmartBAN MAC specifications. In addition, the discovery mechanism is further applicable to other similar types of networks for the discovery of their corresponding neighbour networks.

The rest of the paper is organised as follows. Section II provides a short introduction to the SmartBAN network and details the description on the system model for the proposed neighbour WBAN discovery mechanism. In Section III, neighbour WBAN discovery probability and discovery time are analytically derived. Results are presented in Section IV, and Section V concludes the paper.

\section{System model}

Firstly, the ETSI SmartBAN network is introduced in this section. Secondly, we provide a detailed description of the proposed neighbour wireless body area network discovery mechanism.

\subsection{ETSI SmartBAN network}

A SmartBAN network operates in two physical channels: a control channel $(\mathrm{CCH})$ and a data channel (DCH). The frequency of operation falls within 2401-2481 MHz in the industrial, scientific, and medical (ISM) band. Using $2 \mathrm{MHz}$ channels the system has 3 CCHs and 37 DCHs, from which the utilised channels can be selected based on interference and other possible restrictions.

The $\mathrm{CCH}$ is only utilised for network initialisation and transmitting periodic control channel beacons (C-Beacons) by the coordinator of a SmartBAN, called Hub. Data transmissions are carried out in the $\mathrm{DCH}$, where the Hub transmits a periodic data channel beacon (D-Beacon). The period between two consecutive D-Beacons is termed as Inter-Beacon Interval (IBI), which is conceptually divided into three parts: Scheduled Access Period, Control and Management Period, and Inactive Period. Each of the periods is further divided into time slots $\left(T_{s}\right)$ of equal duration, as depicted in Fig. 1. The Scheduled Access Period and the Control and Management Period together form the 
Active Period, during which devices other than the Hub of the SmartBAN network are allowed to transmit.

Detailed specifications of the SmartBAN network are defined in [2] and [3]. An overview of the SmartBAN PHY and MAC specifications are provided in [7] and [8], respectively, and an overview of the SmartBAN standardisation activities is carried out in [9].

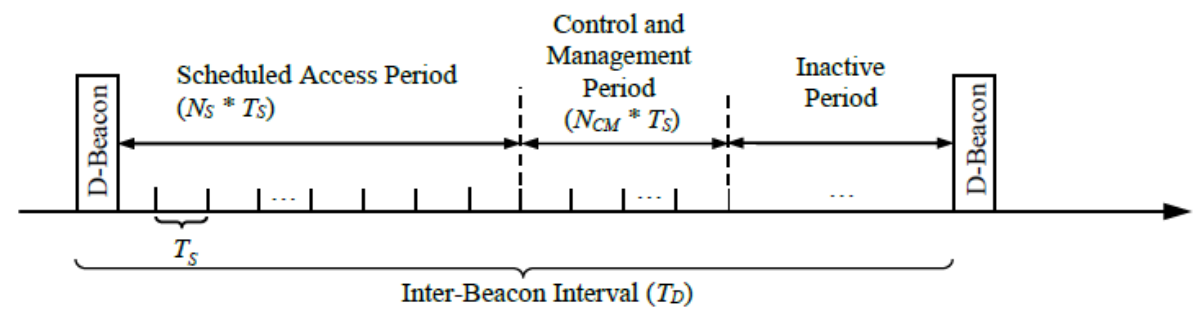

Fig. 1. Inter-Beacon Interval structure of the SmartBAN.

\subsection{Neighbour wireless sensor network discovery mechanism}

The operation of the neighbour WBAN discovery mechanism is based on channel scan performed by the Hub. The scan is executed during the Inactive Periods of the discovering SmartBAN and the aim of the scan is to capture a C-Beacon of a neighbouring SmartBAN. A key factor in the proposed mechanism is the alternation of the Inactive Period durations of the SmartBAN conducting the discovery.

A flowchart of the proposed neighbour wireless body area network discovery mechanism is proposed in Fig. 2. The flowchart includes the following steps in the discovery process.

1. A Hub initiates the discovery mechanism by a higher layer protocol entity issuing hub-to-hub $(\mathrm{H} 2 \mathrm{H})$ discovery start. A Hub actively supporting $\mathrm{H} 2 \mathrm{H}$ communications increases its $\mathrm{C}$-Beacon rate to at least the rate of D-beacon interval $\left(T_{\mathrm{D}}\right) / 2$.

2. The Hub begins issuing an alternating inactive period duration in every forthcoming IBI. Note that $T_{D}$ alternates correspondingly in every IBI.

3. During the first Inactive Period following commencement of the neighbour discovery mechanism the Hub initiates a timer. While the timer has not expired the Hub scans for C-Beacons of other networks during the Inactive Periods. If the timer has expired after the end of inactive period $j$, the Hub resets the timer and starts scanning $\mathrm{C}$-Beacons on the next $\mathrm{CCH}$ of the applicable $\mathrm{CCH}$ list. Once the $\mathrm{CCH}$ list has been exhausted, the Hub stops scanning for $\mathrm{C}$-Beacons and reports the list of neighbour $\mathrm{C}$-Beacons found to the higher layer. If the list is empty, the report indicates a failure.

4. If a C-Beacon or C-Beacons are successfully received during an Inactive Period $j$, the Hub appends those beacons to the neighbour Hub list. Once such an event occurs the Hub may terminate the neighbour network discovery mechanism early (e.g., if all predefined C-Beacons have been found) and report the list to the higher layer. 


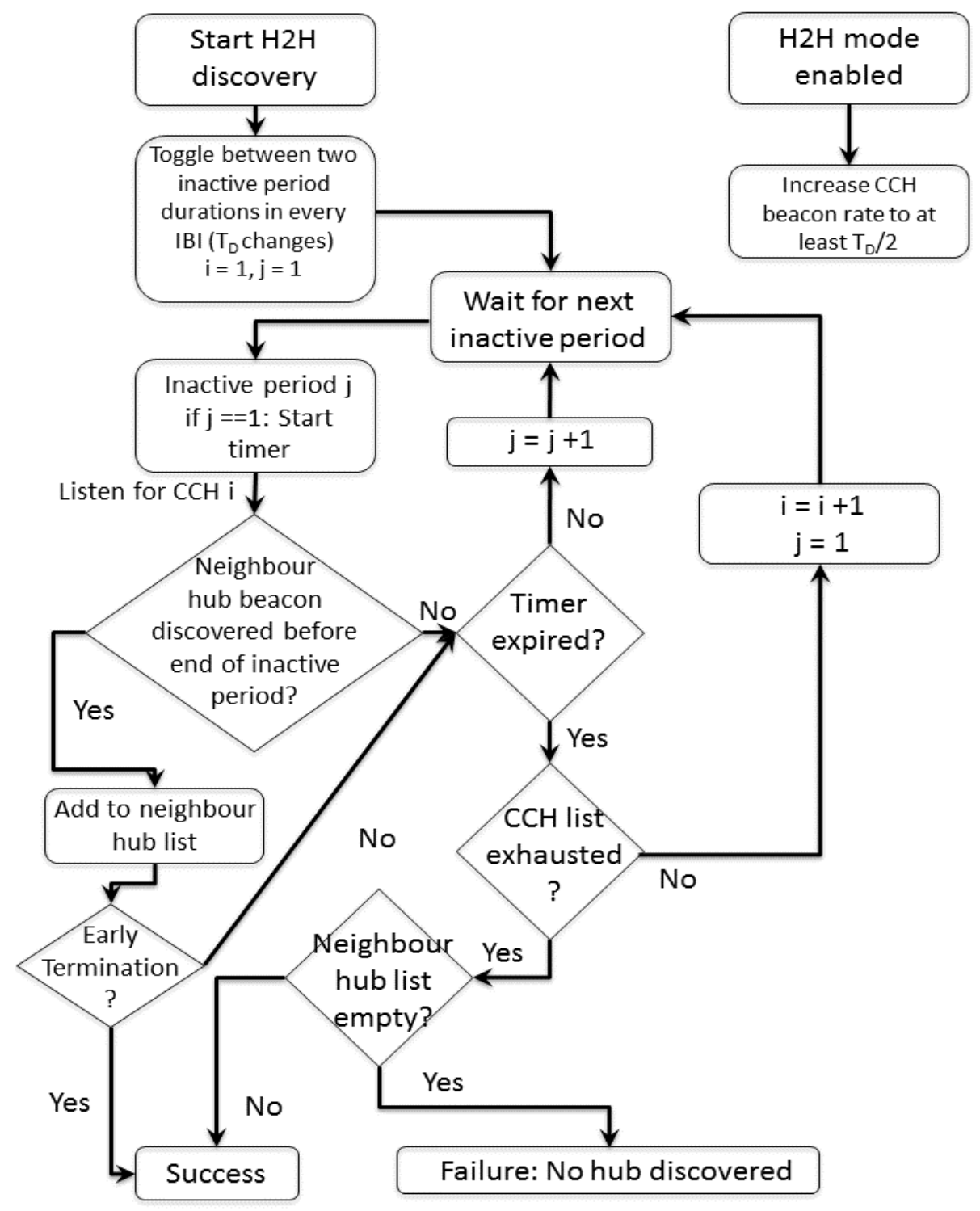

Fig. 2. Flowchart of neighbour wireless body area network discovery mechanism. 


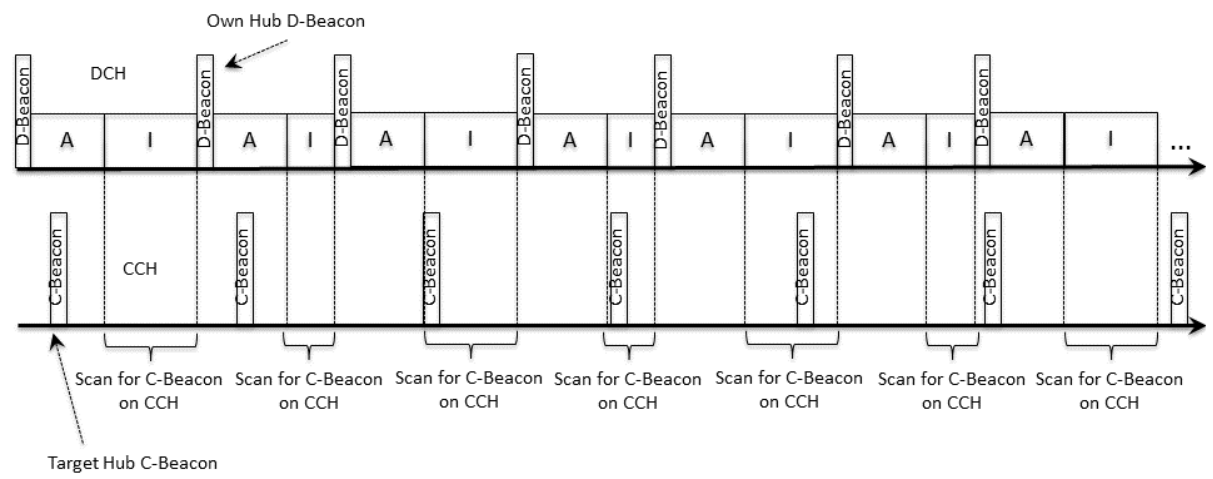

Fig. 3. Example of the effect of alternating inactive period and $T_{\mathrm{D}}$ duration for C-Beacon discovery.

An example on the effect of alternating the inactive period and the $T_{D}$ on C-Beacon discovery is illustrated in Fig. 3. C-Beacon scans in constant length inactive periods may not be able to receive transmitted C-Beacons since a neighbour SmartBAN CBeacon period is unknown to the Hub before discovery. Alternating the inactive period improves the probability of receiving any periodic C-Beacon transmissions as the varying length of the $T_{D}$ disrupts the constant cyclic transmission of D-Beacons and shifts the relative occurrence of the inactive period scans with respect to the constant cyclical C-Beacon transmissions.

It should also be noted that the proposed model provides a mechanism for the discovery of other neighbouring networks without interrupting the on-going operations of either the wireless network carrying out the discovery or the wireless networks to be discovered. Current methods either do not provide for such a mechanism or they require the shutdown of the existing discovering network and its re-establishment as a child network of another wireless network after such network's discovery and association to it. The discovery mechanism is originally presented in [10].

\section{Analysis}

This section proposes a mathematical analysis for the proposed neighbour WBAN discovery mechanism. The analysis is carried out in terms of probability of discovery and discovery time for both the non-alternating and the alternating Inactive Periods.

\subsection{Probability of discovery}

Firstly, we define the probability of discovery in a case where non-alternating Inactive Period durations are utilised, i.e. Inter-beacon Interval remains constant. Secondly, the probability of discovery is defined for the proposed mechanism having alternating Inactive Period durations. 
Non-alternating Inter-Beacon Interval. We define a maximum time, $T_{\text {Scan }}$, for the $\mathrm{CCH}$ scan. Based on $T_{\mathrm{Scan}}$, the number of Inter-Beacon Intervals during the maximum $\mathrm{CCH}$ scan time is

$$
N_{I B I}=\left\lfloor\frac{T_{S c a n}}{T_{D}}\right\rfloor
$$

The probability of a C-Beacon transmission of a neighbouring SmartBAN during a single time slot is

$$
P_{C B \mid T S}=\frac{T_{S}}{T_{C}},
$$

where $T_{\mathrm{S}}$ is time slot duration, and $T_{\mathrm{C}}$ is the C-Beacon interval. Correspondingly, the probability of not having a $\mathrm{C}$-Beacon transmission during a single time slot is

$$
P_{N O C B \mid T S}=1-\frac{T_{S}}{T_{C}} \text {. }
$$

The probability of being in an Inactive Period of the discovering SmartBAN is

$$
P_{I}=\frac{N_{S l o t}{ }_{I B I}-N_{S l o t}{ }^{-1}}{N_{S l o t}},
$$

where $N_{\text {Slot_IBI }}$ is the number of slots in the IBI, $N_{\text {Slot_A }}$ is the number of slots in the Active Period, and -1 represents the Beacon slot. Based on (3) and (4) the probability of not having at least a single $\mathrm{C}$-Beacon during a single Inactive Period is

$$
P_{N o C B \mid I}=\left(1-\frac{T_{S}}{T_{C}}\right)^{N_{S l o t}{ }_{I B I}-N_{S l o t}{ }_{A}^{-1}}
$$

Therefore, the probability of having at least a single C-Beacon during the Inactive Period is

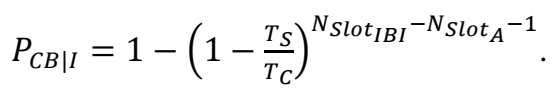

Finally, the probability of having a C-Beacon during one of the Inactive Periods during the maximum $\mathrm{CCH}$ scan time is

$$
P_{C}=1-\left(1-P_{I} P_{C B \mid I}\right)^{N_{I B I}}
$$

Alternating Inter-Beacon Interval. The probability of discovery for the proposed mechanism using alternating Inactive Period durations is calculated similarly. The number of alternating tuple Inter-Beacon Intervals during the maximum $\mathrm{CCH}$ scan time is

$$
N_{I B I_{-} A l t}=\left\lfloor\frac{T_{S c a n}}{T_{D}+T_{D 2}}\right\rfloor,
$$

where $T_{\mathrm{D} 2}$ is the length of the increased Inter-Beacon Interval, $\mathrm{IBI}_{2}$. The number of either normal or increased IBIs during the maximum $\mathrm{CCH}$ scan time is 


$$
N_{\text {IBI_Comb }}=\left\lfloor\frac{T_{S c a n}}{\frac{T_{D}}{T_{D}+T_{D 2}} T_{D}+\frac{T_{D 2}}{T_{D}+T_{D 2}} T_{D 2}}\right\rfloor .
$$

The probability of being in an increased Inactive Period during $\mathrm{IBI}_{2}$ of the discovering SmartBAN is

$$
P_{I 2}=\frac{N_{S l o t}{ }_{I B I 2}-N_{S l o t}-1}{N_{\text {Slot }}{ }_{I B I 2}}
$$

where $N_{\text {Slot_IBI2 }}$ is the number of slots in $\mathrm{IBI}_{2}$. The probability of having a C-Beacon during the increased Inactive Period is

$$
P_{C B \mid I 2}=\left(1-\frac{T_{S}}{T_{C}}\right)^{N_{S l o t} I B I 2-N_{S l o t}{ }^{-1}} .
$$

Based on (7) - (11), the probability of having a C-Beacon during one of the Inactive Periods (normal or increased) during the maximum $\mathrm{CCH}$ scan time is

$$
P_{C 2}=1-\left(1-\frac{T_{D}}{T_{D}+T_{D 2}} P_{I} P_{C B \mid I}+\frac{T_{D 2}}{T_{D}+T_{D 2}} P_{I 2} P_{C B \mid I 2}\right)^{N_{I B I} C o m b}
$$

\subsection{Discovery time}

Non-alternating Inter-Beacon Interval. The C-Beacon discovery time for non-alternating Inter-Beacon Interval scan is derived from Fig. 4, where $T_{\mathrm{A}}$ and $T_{\mathrm{I}}$ are the durations of Active Period and Inactive Period, respectively. $\mathrm{T}_{\mathrm{C}_{-} \text {offset }}$ is the offset between a D-Beacon of the discovering SmartBAN, and a C-Beacon of the target SmartBAN. The C-Beacon is discovered if the inequality

$$
n T_{A}+(n-1) T_{I} \leq T_{C_{O f f s e t}}+k T_{C}<n T_{D}, \forall n \in 1,2,3 \ldots, \forall k \in 0,1,2 \ldots
$$

holds, where the maximum value of $n=N_{\mathrm{IBI}}$, and $\mathrm{k}$ is an integer with a maximum value of

$$
k=\left\lceil\frac{T_{S c a n}}{T_{C}}\right\rceil .
$$

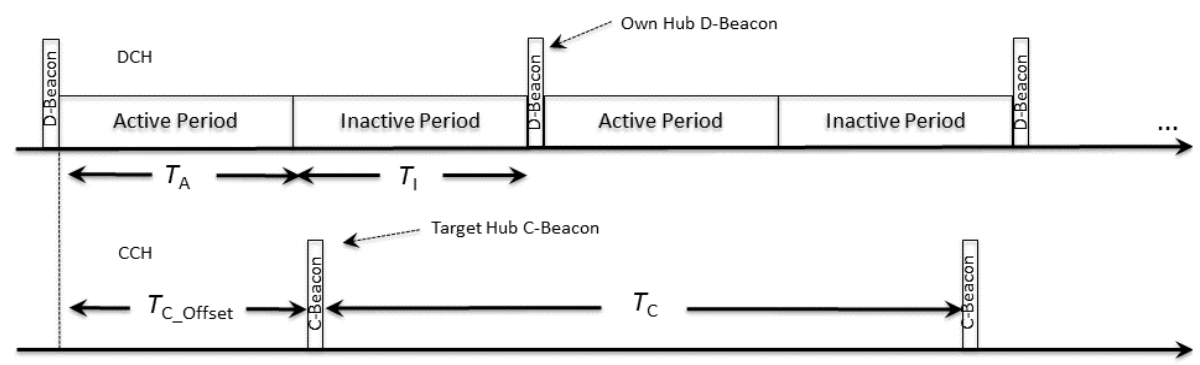

Fig. 4. C-Beacon discovery time for non-alternating Inter-Beacon Interval. 
Alternating Inter-Beacon Interval. The C-Beacon discovery time for alternating Inter-Beacon Interval scan is derived from Fig. 5, where $T_{12}$ is the duration of the Increased Inactive Period. The $\mathrm{C}$-Beacon is discovered if either of the inequalities

$n T_{A}+(n-1)\left(T_{I}+T_{I 2}\right) \leq T_{C_{O f f s e t}}+k T_{C}<n\left(T_{\mathrm{A}}+T_{I}\right)+(n-1)\left(T_{A}+T_{I 2}\right), \forall n \in$

$1,2,3 \ldots, \forall k \in 0,1,2 \ldots$

or

$n\left(2 T_{A}+\mathrm{T}_{I}\right)+(n-1) T_{I 2} \leq T_{C_{O f f s e t}}+k T_{C}<n\left(2 T_{\mathrm{A}}+T_{I}+T_{I 2}\right), \forall n 1,2,3 \ldots, \forall k \in$

$0,1,2 \ldots$

hold, where the maximum value of $n=N_{\text {IBI_Alt. }}$

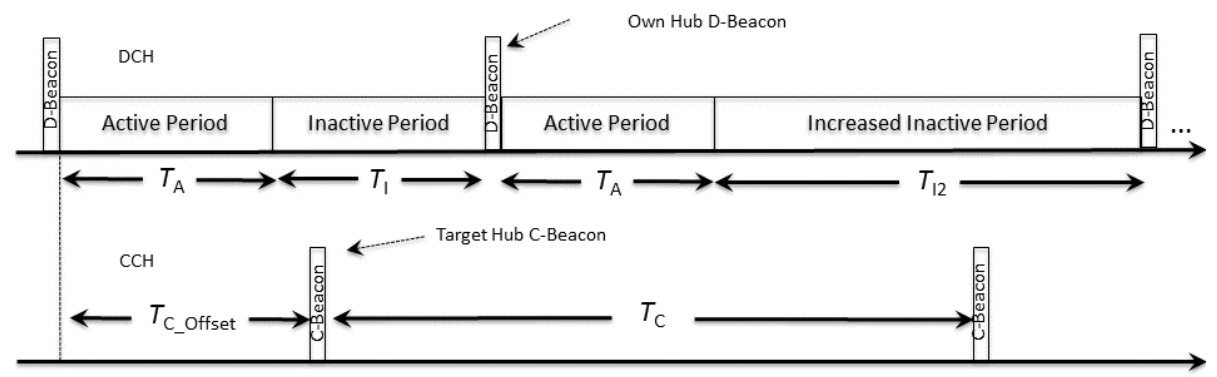

Fig. 5. C-Beacon discovery time for alternating Inter-Beacon Interval.

\section{$4 \quad$ Results}

The most relevant SmartBAN network parameters utilised in the performance analysis are presented in Table 1.

Control Channel Beacon discovery probability as a function of Data Channel InterBeacon Interval and Control Channel Inter-Beacon Interval is presented in Fig. 6. The proposed discovery mechanism utilising alternating Inter-Beacon Interval durations outperforms the reference mechanism using non-alternating Inter-Beacon Interval durations in almost all cases. The discovery probability is 1 for the proposed mechanism when Data Channel Inter-Beacon Interval is $0.5 \mathrm{~s}$ or less, and Control Channel InterBeacon Interval is $0.2 \mathrm{~s}$ or less. Since the current SmartBAN specifications support up to 16 nodes in a SmartBAN network, the above-mentioned limit for the Data Channel Inter-Beacon Interval can be considered feasible. With the utilised $0.00125 \mathrm{~s}$ time slot length, and $50 \%$ duty cycle of the network, one time slot can be allocated to 16 nodes in every Inter-Beacon Interval, and still nine time slots remain to be used by the Control 
Table 1. SmartBAN network parameters.

\begin{tabular}{ll}
\hline Parameter & Value \\
\hline Time Slot Length $\left(T_{\mathrm{S}}\right)$ & $0.00125 \mathrm{~s}$ \\
D-Beacon Interval $\left(T_{\mathrm{D}}\right)$ & $0.1,0.2,0.5,0.8,1.0,1.5,2.0,2.5,3.0,5.0 \mathrm{~s}$ \\
C-Beacon Interval $\left(T_{\mathrm{C}}\right)$ & $0.01,0.1,0.2,0.5,0.8,1.0,1.5,2.0,2.5,3.0 \mathrm{~s}$ \\
Control Channel Beacon offset as fraction & $0,0.2 T_{\mathrm{C}}, 0.5 T_{\mathrm{C}}, 0.8 T_{\mathrm{C}}$ \\
of $T_{\mathrm{C}}(\mathrm{CCH}$ fffset $)$ & 2 \\
Inactive Period Increase Factor & $5.0 \mathrm{~s}$ \\
Maximum Scan Time $\left(T_{\mathrm{Scan}}\right)$ & \\
\hline
\end{tabular}

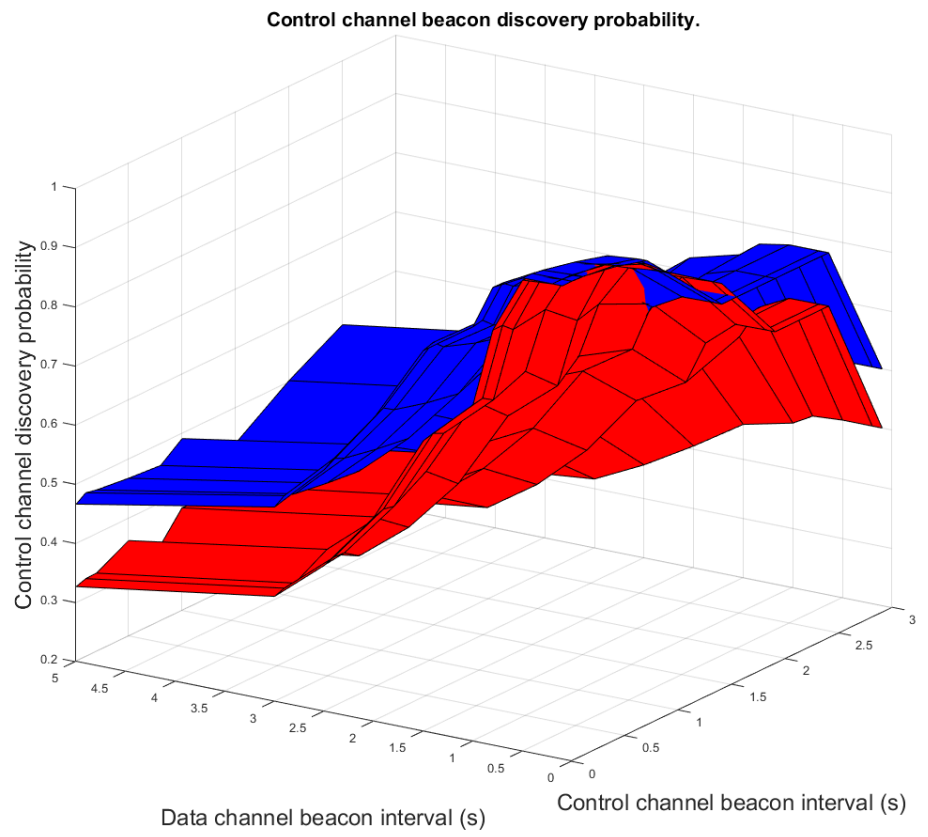

Fig. 6. Control Channel Beacon discovery probability as a function of Data Channel Inter-Beacon Interval and Control Channel Inter-Beacon Interval.

and Management Period of the Inter-Beacon Interval.

When averaged over the entire data set, the alternating mechanism provides, on average, $18 \%$ higher C-Beacon discovery probability as compared to the non-alternating mechanism. At best, alternating mechanism provides $61 \%$ higher discovery probability (Data Channel Inter-Beacon Interval $=5 \mathrm{~s}$, Control Channel Inter-Beacon Interval $=2 \mathrm{~s}$ ). In addition, in $89 \%$ of the cases the alternating mechanism provides better discovery 
probability as compared to the non-alternating version, and in $3 \%$ of the cases equal probability.

When the Data Channel Inter-Beacon Interval, and/or the Control Channel InterBeacon Interval increase, the discovery probability decreases. The results are expected, since in those cases there are less Inactive Periods (i.e. less individual scans), and less Control Channel Beacon transmissions. Therefore, the discovery probability becomes heavily dependent on the timings of the neighbouring networks. In a worst case scenario, the Data Channel Inter-Beacon Interval duration of the discovering network and the Control Channel Inter-Beacon Interval duration of the neighbouring network are the same, and Beacon transmissions occur at the same time. In this case, the discovery probability would be zero with the reference mechanism.

The Control Channel Beacon discovery time as a function of Data Channel InterBeacon Interval and Control Channel Inter-Beacon Interval with Control Channel Beacon offset $=0,0.2,0.5$, and 0.8 is presented from Fig. 7 through Fig. 10, respectively. In the figures a value of $5.5 \mathrm{~s}$ on the discovery time indicates the Control Channel Beacon could not be found during the maximum scan time. Based on the figures it can be stated that the proposed discovery mechanism utilizing alternating Inter-Beacon Interval durations outperforms the reference mechanism using non-alternating Inter-Beacon Interval durations in almost all cases in terms of discovery time as well. When considering Data Channel Inter-Beacon Interval and Control Channel Inter-Beacon Interval durations of $0.5 \mathrm{~s}$ or less, and Control Channel Beacon offset 0 , the proposed mechanism captures Control Channel Beacon approximately in $0.2 \mathrm{~s}$, whereas the reference mechanism captures it approximately in $0.4 \mathrm{~s}$. The effect of Control Channel Beacon offset on the discovery is clearly visible, when utilising the reference discovery mechanism. Even with short Data Channel Inter-Beacon Interval and Control Channel InterBeacon Interval durations there are cases in which the Control Channel Beacon cannot be discovered. Since the relative occurrence of the Inactive Period, i.e. the scan, remains constant in this case, the result is expected. When utilising the proposed mechanism, the aforementioned phenomenon is mostly avoided. 


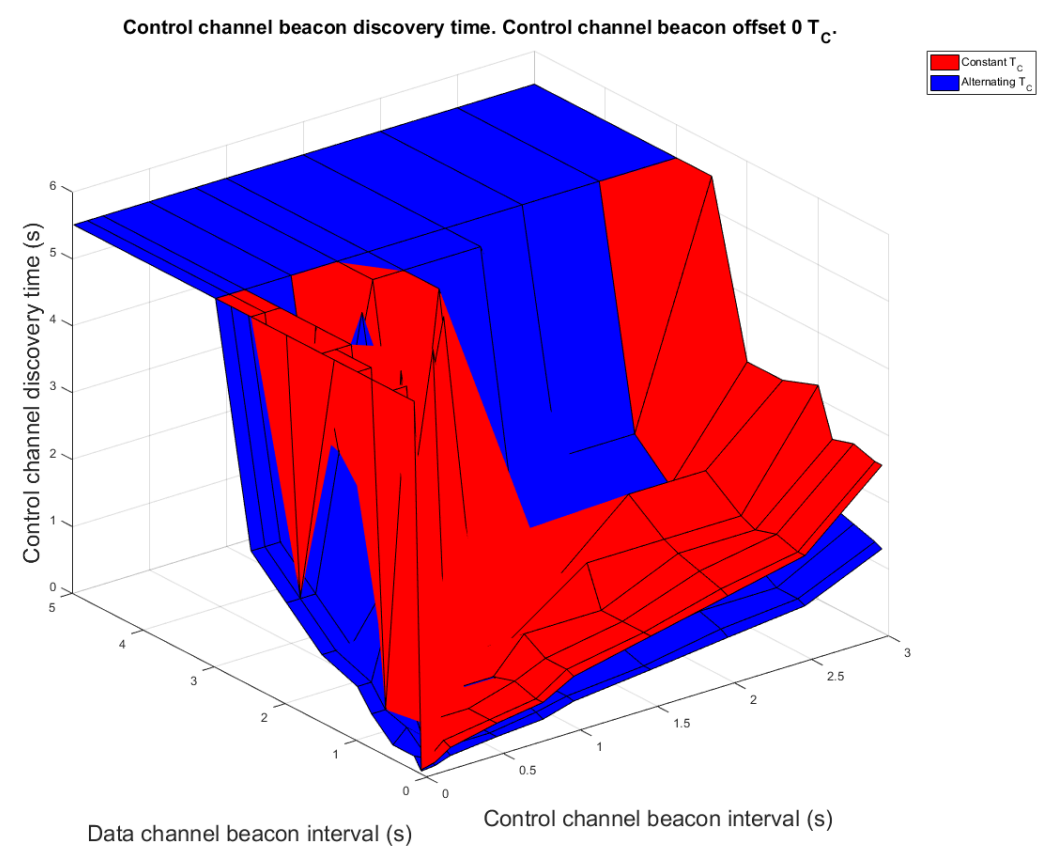

Fig. 7. Control Channel Beacon discovery time as a function of Data Channel Inter-Beacon Interval and Control Channel Inter-Beacon Interval. Control Channel Beacon offset $=0$. A value of $5.5 \mathrm{~s}$ indicates failure to discover a C-Beacon. 


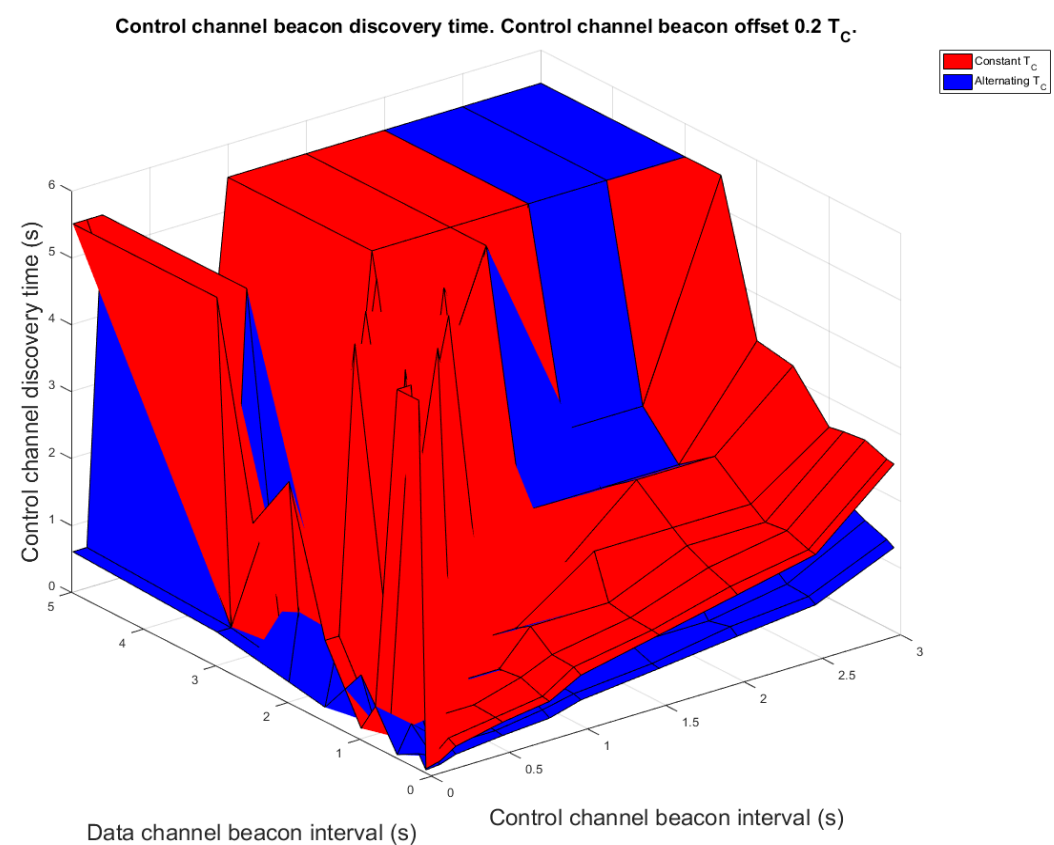

Fig. 8. Control Channel Beacon discovery time as a function of Data Channel Inter-Beacon Interval and Control Channel Inter-Beacon Interval. Control Channel Beacon offset $=0.2$. A value of $5.5 \mathrm{~s}$ indicates failure to discover a $\mathrm{C}$-Beacon. 


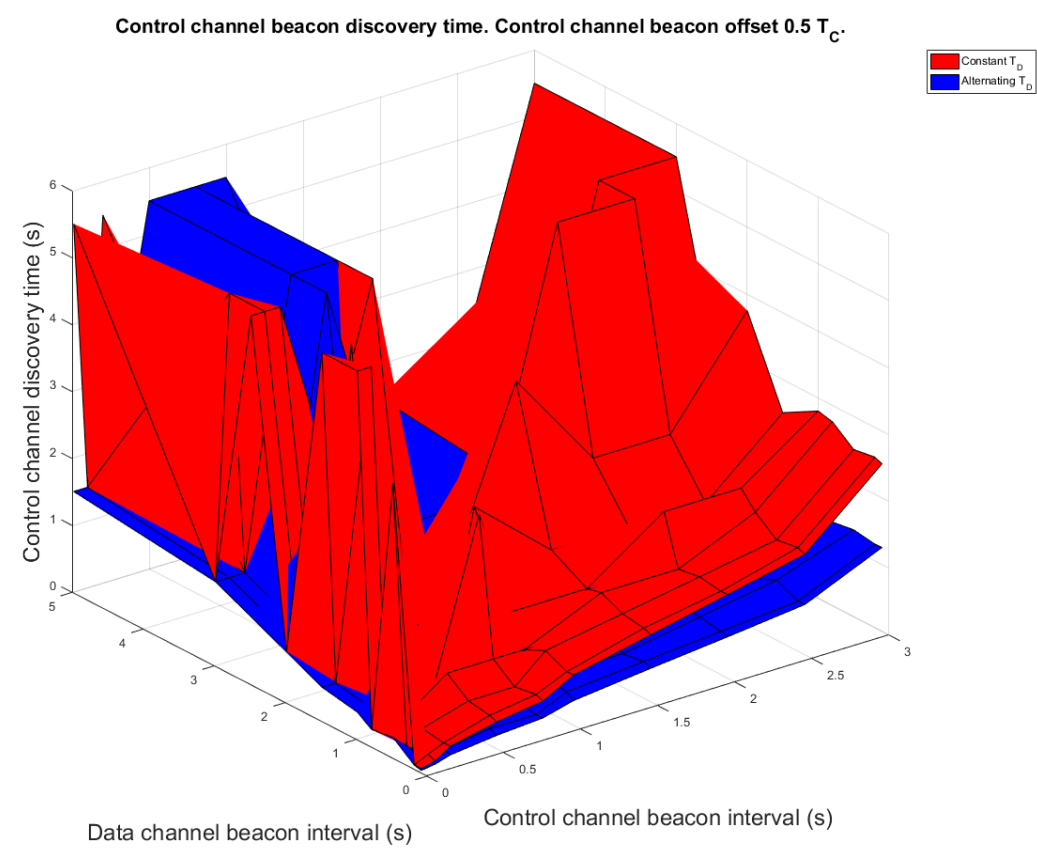

Fig. 9. Control Channel Beacon discovery time as a function of Data Channel Inter-Beacon Interval and Control Channel Inter-Beacon Interval. Control Channel Beacon offset $=0.5$. A value of $5.5 \mathrm{~s}$ indicates failure to discover a $\mathrm{C}$-Beacon. 


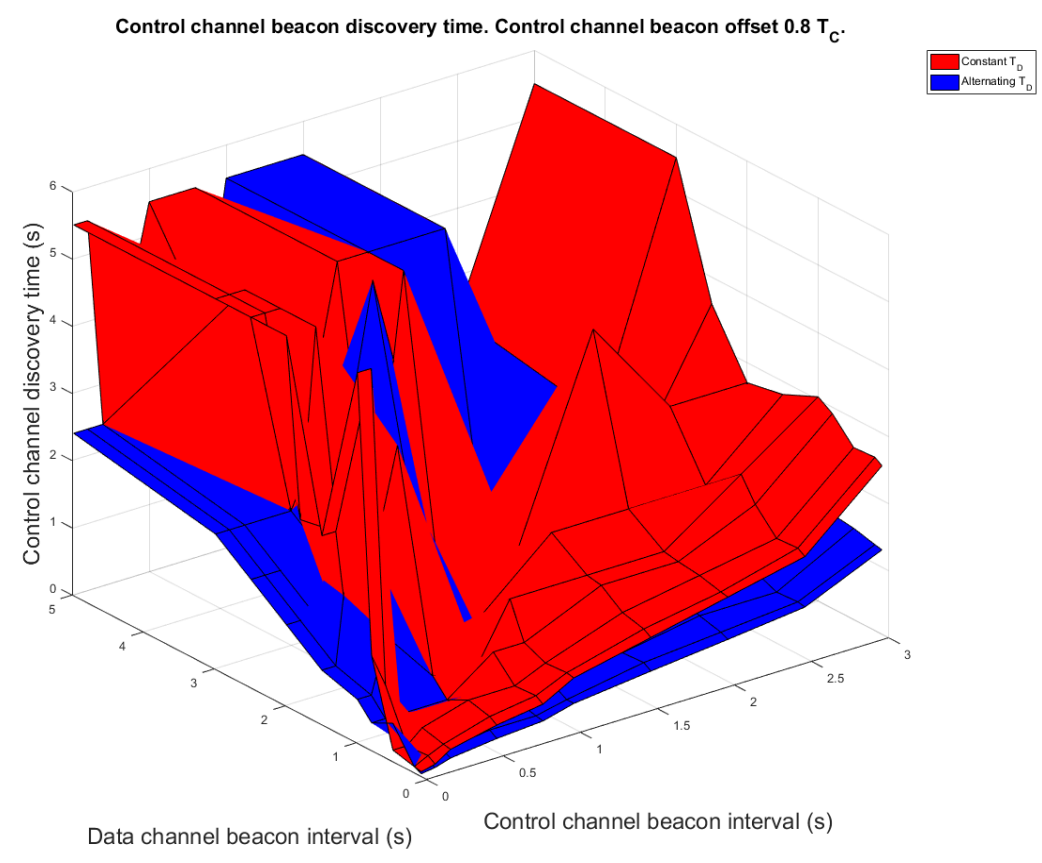

Fig. 10. Control Channel Beacon discovery time as a function of Data Channel Inter-Beacon Interval and Control Channel Inter-Beacon Interval. Control Channel Beacon offset $=0.8$. A value of $5.5 \mathrm{~s}$ indicates failure to discover a C-Beacon.

\section{Conclusion}

In this paper, we introduced a novel neighbour network discovery mechanism for WBANs. The mechanism is based on the utilisation of the Inactive Periods of the discovering WBAN by alternating the Inactive Period durations for scanning neighbouring WBANs. The key advantage of the mechanism is that it is defined for the case where a WBAN is already operational and attempts to discover another operational WBAN without interrupting either WBAN operations already being carried out. Furthermore, the mechanism is SmartBAN compliant, and currently it is under consideration to be included in the ongoing revision of SmartBAN MAC specifications.

We have analytically derived the performance of the proposed mechanism in terms of discovery probability, and discovery time. Based on the results it can be concluded that the proposed mechanism outperforms a reference mechanism using non-alternating Inactive Period durations for the discovery in almost all cases. The main reason for better performance is that alternating the Inactive Period improves the probability of receiving any periodic C-Beacon transmissions, since the varying length of the InterBeacon Interval disrupts the constant cyclic transmission of D-Beacons. Therefore, it shifts the relative occurrence of the Inactive Period scans with respect to the constant cyclical C-Beacon transmissions. 
Based on the results it can also be stated that in certain special cases neighbour network cannot be discovered. However, these cases can be solved by provider specific implementation in defining network parameters.

\section{Acknowledgment}

This research has been financially supported in part by Academy of Finland 6Genesis Flagship (grant 318927).

\section{References}

1. IEEE Computer Society, LAN/MAN Standards Committee: IEEE Std 802.15.6-2012, IEEE Standard for Local and metropolitan area networks - Part 15.6: Wireless Body Area Networks. IEEE, (2012).

2. ETSI TC SmartBAN: Smart Body Area Network (SmartBAN), Enhanced Ultra-Low Power Physical Layer, ETSI TS 103326 V1.1.1. ETSI (2015).

3. ETSI TC SmartBAN: Smart Body Area Network (SmartBAN), Low Complexity Medium Access Control (MAC) for SmartBAN, ETSI TS 103325 V1.1.1. ETSI (2015).

4. Sun, W., Yang, Z., Zhang, X., Liu, Y.: Energy-Efficient Neighbor Discovery in Mobile Ad Hoc and Wireless Sensor Networks: A Survey. IEEE Communications Surveys \& Tutorials 16 (3), 1448-1459 (2014).

5. Liu, J., Chen, C., Ma, Y.: Modeling neighbor discovery in Bluetooth Low Energy Networks. IEEE Communications Letters 6 (9), 1439-1441 (2012).

6. Jeon, W.S., Wwijaksara, M.H., Jeong, D.G.: Performance Analysis of Neighbor Discovery Process in Bluetooth Low-Energy Networks. IEEE Transactions on Vehicular Technology 66 (2), 1865-1871 (2017).

7. Chin, W.H., Tanaka, H., Nakanishi, T., Paso, T., Hämäläinen, M.: An Overview of ETSI TC SmartBAN's Ultra Low Power Physical Layer. In: 9th International Symposium on Medical Information and Communication Technology, IEEE, Kamakura, Japan (2015).

8. Paso, T., Tanaka, H., Hämäläinen, M., Chin, W.H., Matsuo, R., Subramani, S., Haapola, J.: An Overview of ETSI TC SmartBAN MAC Protocol. In: 9th International Symposium on Medical Information and Communication Technology, IEEE, Kamakura, Japan (2015).

9. Hämäläinen, M., Paso, T., Mucchi, L., Girod-Genet, M., Farserotu, J., Tanaka, H., Chin, W.H., Nachabe Ismail, L.: ETSI TC SmartBAN - Overview of the Wireless Body Area Network Standard. In: 9th International Symposium on Medical Information and Communication Technology, IEEE, Kamakura, Japan (2015).

10. Paso, T., Haapola, J.: Neighbour Wireless Sensor Network Discovery Mechanism. Patent Application FI 20175994 (2017). 